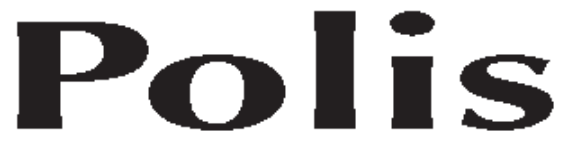

\title{
Biocombustibles: ¿una estrategia de desarrollo o de mercado lucrativamente sostenible?
}

\author{
Lazaro Camilo Recompensa Joseph* \\ Benedito Dias Pereira** \\ Arturo Zabala Zabala \\ Alexandre de Melo Farias*** \\ Pedro Ramos****
}

\begin{abstract}
Resumen: El trabajo tiene como objetivo analizar la incompatibilidad teórica existente entre el concepto (y/o los principios) del desarrollo sostenible y la producción de biocombustibles fundamentada en el modelo del agronegocio o agribusiness. Así en el primer ítem describimos los principales alertas emitidos a través de los Informes del IPCC (Intergovernmental Panel on Climate Change, o Panel Inter gubernamental sobre los Cambios Climáticos) destacando: a) la influencia y el papel de las actividades humanas en el proceso del calentamiento global y la contaminación ambiental, y b) el costo de oportunidad de reducir las emisiones de $\mathrm{CO}_{2}$. En el segundo destacamos los principales inversionistas extranjeros interesados en la producción de los biocombustibles, colocando algunas reflexiones en relación a este negocio "lucrativamente sostenible", y por último -a modo de consideración- enfatizamos la imposibilidad en relación a la creencia absoluta en el libre mercado como la mejor fuente de innovación y aprendizaje para desarrollar alternativas energéticas viables y sostenibles ambientalmente.

Palabras claves: Biocombustíbles y desarrollo sostenible, Agronegocio, Agro energía y libre mercado, desarrollo local, economía agrícola.
\end{abstract}

\section{Biofuels: a strategy for development or for a sustainable lucrative market}

Abstrat: This paper aims to analyze theoretical existing incompatibilities between the concept (and / or principles) of sustainable development and biofuels production based on the agribusiness model. In the first item we describe the main alerts issued through the IPCC (Intergovernmental Panel on Climate Change) Report highlighting: a) the influence and role of human activities in the global warming process and environmental pollution, and $b$ ) the cost of

*Universidade Federal de Mato Grosso, Cuiaba, Brasil. Email: camilojoseph@ hotmail.com

${ }^{* *}$ Universidade Federal de Mato Grosso, Cuiaba, Brasil. Email: bdp@terra.com.br

*** Universidade Federal de Mato Grosso, Cuiaba, Brasil. Email: arturoz@ig.com.br

**** Universidade Federal de Mato Grosso, Cuiaba, Brasil. Email: melofaria@ hotmail.com

${ }^{* * * * *}$ Universidade Estadual de Campinas, Campina, Brasil. Email: peramos@eco.unicamp.br 
opportunity in reducing $\mathrm{CO} 2$ emissions. In the second item we point out the major foreign investors interested in biofuels production, placing some reflections regarding this "profitably sustainable" business. As last consideration, we deny the absolute belief on free market as the best source for innovation and learning in order to develop viable and environmentally sustainable energetic alternatives.

Key words: Biofuels and sustainable development, Agribusiness, Agri energy and free market, local development, agricultural economy.

\section{Introducción}

El mundo se encuentra frente a una enorme campaña global, cuyo objetivo es incorporar de la forma más rápida posible diferentes materias primas tales como: caña de azúcar, soya, maíz, colza, remolacha, etc., a la producción de biocombustíbles como substitutos perfectos de los derivados de petróleo. Las principales justificaciones encontradas para este fenómeno tienen su fundamento en el calentamiento global y en la contaminación del medio ambiente.

O sea, se pretende sustituir todo el consumo de energía fósil (petróleo) utilizando los biocombustíbles, producidos bajo la influencia del modelo de agronegocio o agribusiness, modelo de producción agropecuario que ha aportado y suministrado "magníficas" cosechas, y que posee un conjunto de particularidades tales como: a) altísimo consumo de energía fósil, b) necesidad de utilizar grandes establecimientos agropecuarios y concentración de tierras c) uso intensivo de tecnologías y máquinas en detrimento del trabajo humano, d) daños ambientales en gran escala, e) alto consumo de agua en los cultivos irrigados, f) presencia de modificaciones genéticas para el mejoramiento de semillas y rebaños, g) alta concentración de capitales (aunque se conozca que la mayoría de esas materias primas que serán utilizadas para esos objetivos presentan bajo balance energético, entendido como la relación existente entre el gasto de energía fósil utilizado para obtener una unidad de biodiesel y la energía obtenida en forma de biodiesel).

En este sentido debemos destacar las diferencias entre la energía que se pretende extraer hoy de la agricultura y la contenida en la energía fósil (petróleo, gas, natural y carbón) que representa el $80 \%$ de la energía consumida en el mundo. O sea, la primera se obtiene como resultado de la fotosíntesis: el sol, el agua y los nutrientes que a cada año se adquieren con las cosechas, aunque la energía fósil tiene el mismo origen pero con la diferencia de que es resultado del ahorro de la fotosíntesis producida a lo largo de millones de año. En síntesis se espera que la naturaleza nos entregue a cada año, el equivalente ahorrado durante esos millones de años.

Planteado así el problema podemos afirmar que el biodiesel y la biomasa no podrán 
actuar como sustitutos energéticos perfectos, porque la fotosíntesis que los origina es limitada y tendrán que ser utilizados prioritariamente como sustitutos en la producción actual de los fertilizantes y de la actual mecanización agrícola pilar fundamental del modelo de Agronegocio.

En realidad las principales economías del mundo, que son las mayores contaminadoras del planeta, están realizando una alianza en la cual participan las multinacionales petroleras, biotecnológicas, montadoras de auto, los grandes productores de granos, bancos, etc., siendo sus agentes los que decidirán el destino y el uso de las tierras, los paisajes rurales de algunas regiones de América Latina.

Este trabajo tiene como objetivo analizar la incompatibilidad teórica existente entre el concepto (y/o los principios) de desarrollo sostenible y la producción de biocombustibles basada en el modelo del agronegocio o agribusiness. Así en el primer punto del trabajo describimos los principales alertas emitidos a través de los Informes del IPCC (Intergovernmental Panel on Climate Change, o Panel Inter gubernamental sobre los Cambios Climáticos) sobre: a) la influencia y el papel de las actividades humanas en el proceso del calentamiento global y la contaminación ambiental, b) el costo de oportunidad de reducir las emisiones de $\mathrm{CO}_{2}$; en el segundo ítem se analiza los principales inversionistas extranjeros interesados en la producción de los biocombustibles, presentándose algunas reflexiones en relación a este negocio "lucrativamente sustentable".

\section{Desarrollo sostenible: el boom del etanol y de los biocombustibles a la luz de los cambios climáticos: El remedio puede ser peor que la enfermedad}

La primera parte del cuarto informe de la evaluación de la salud de la atmósfera, elaborado por el IPCC, es preciso y contundente al no dejar dudas de que la mayor parte del calentamiento de los últimos 50 años se debe exactamente a las emisiones de los gases de efecto estufa provocadas y generadas por las actividades humanas.

Según el IPCC $^{1}$ la concentración inédita de gases de efecto invernadero en la atmósfera (dióxido de carbono $\left(\mathrm{CO}_{2}\right.$ y metano) son producidos por la quema de petróleo y derivados, carbón, agricultura y destrucción de las florestas tropicales. Aun así, las emisiones humanas de gases no paran de aumentar. Las mismas crecieron de 6,4 billones de toneladas anuales en 1990 para 7,2 billones en esta década.

El informe mencionado originó y generó la reacción de los diferentes gobiernos del

\footnotetext{
${ }^{1}$ Ver, Intergovernmental Panel on Climate Change Working Group I to the Fourth Assessment Report of the Climate Change 2007: The Physical Science Basis, February, 2007.
} 
planeta, destacándose las palabras del ex presidente francés Jacques Chirac que comentó: «es hora de una revolución en los estándares de producción y consumo de energía». Justo en el momento que el mundo consume más petróleo y el consumo global se encuentra a punto de igualarse con la oferta, en el nivel de 86 millones de barriles por día. Una frase de un anuncio publicitario del grupo norteamericano Chevron Texaco, ilustra el dramático aumento del consumo de petróleo en el planeta en los últimos años al destacar: «Fueron necesarios 125 años para que el mundo consumiese el primer trillón de barriles de petróleo, pero bastaron 30 años para consumir el segundo».

Además la multinacional Exxon, la mayor petrolera del mundo, disfrutó en el año de 2006 de la mayor ganancia de la historia del capitalismo: US\$ 39,5 billones. En cuanto el planeta continúe dependiendo de cifras y objetivos como ese no existirá sosiego y por consiguiente las altas temperaturas y el aumento del nivel del mar continuarán su espiral de ascensión. Por tanto el desafío es saber ¿cómo el mundo va resolver (hasta el 2020 ó 2030) ese problema en un espacio de tiempo tan corto?

Evidentemente es de consenso general, para todos aquellos agentes y actores del desarrollo sean empresarios, políticos, etc. y que asumen una postura pragmática argumentando que el calentamiento global es "simpleza" y que los informes son excesivamente alarmantes, que deben tener presente que en la percepción de la sociedad el concepto de cambios climáticos es un hecho real, ya está presente y se constituye en un proceso irreversible.

Siendo así, el problema apunta hacia el análisis del "costo de oportunidad" de actuar o no actuar, para minimizar este problema. Según Rajendra Pachauri presidente del IPCC, existen dos nuevos conceptos o términos que pasan a dominar el debate internacional sobre el clima: la mitigación (como disminuir emisiones de $\mathrm{CO}_{2}$ o retirarlas de la atmósfera) y la adaptación (proteger a las poblaciones de los efectos inevitables).

Es en el primer campo el de la mitigación que se dan los principales embates políticos. Los países ricos, los que más contribuyen para el calentamiento global, los que más polusionan y contaminan el medio ambiente quieren que las naciones en desarrollo también admitan compromisos de reducción.

El propio informe del IPCC argumenta que una posible solución sería disminuir a la mitad la emisión de $\mathrm{CO}_{2}$ (lo que representa un enorme desafío) y que como veremos a continuación, torna más complicadas y sensiblemente más enredadas las negociaciones entre los países ricos y pobres con la intención de desacelerar el calentamiento global. (Ver gráficos 1 y 2). 


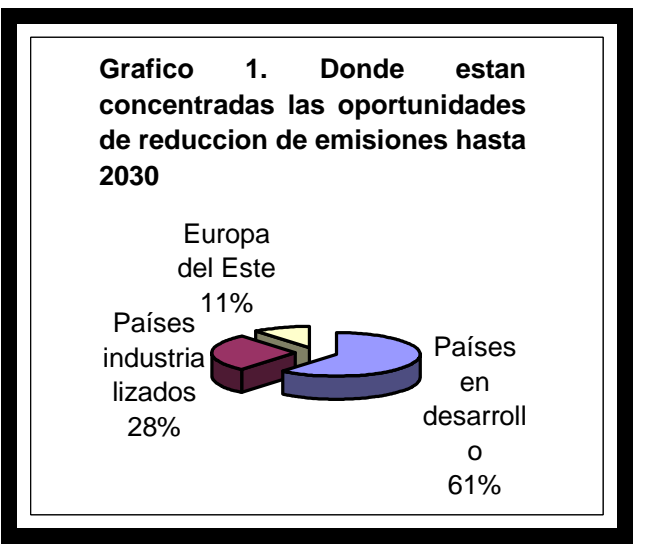

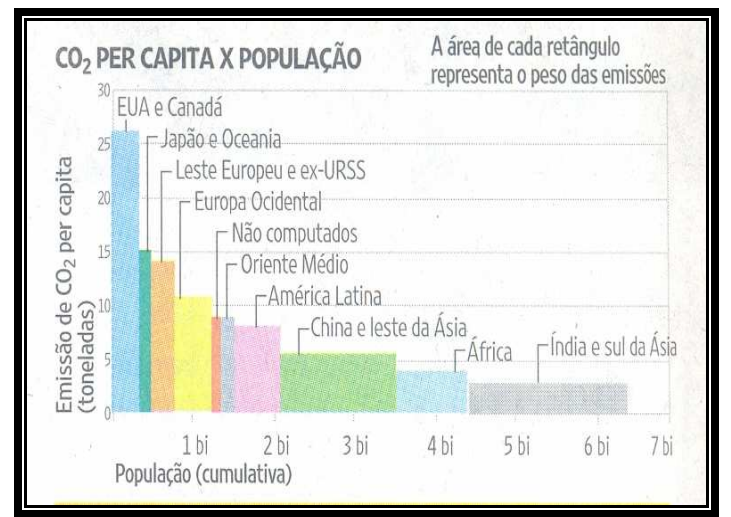

Gráfico no. 2. Emisiones de $\mathrm{CO}_{2}$ per capita en toneladas

Fuente: IPCC Working Group III Fourth Assessment Report. Climate Change 2007: Mitigation of Climate Change. Bangkok Thailand, May 2007.

De los gráficos presentados podemos deducir lo siguiente:

- El mayor volumen de oportunidades mas fáciles para frenar el proceso de cambios climáticos se encuentra en los países pobres, que al mismo tiempo son los que menos consumen, y menos emiten $\mathrm{CO}_{2}$, contaminan y polusionan el medio ambiente. $\mathrm{O}$ sea, detener el calentamiento global será una tarea extremamente difícil y los resultados van a demorar en aparecer y será necesario negociar mucho para que el potencial de reducción en la emisión de gases de invernadero en los países en desarrollo puedan ser aprovechadas.

- Según Meinrat Andreae, climatólogo del Instituto Max Planck de Química de Alemania, para intentar disminuir el calentamiento global en $2^{\circ} \mathrm{C}$ deben ser evacuadas desde la atmósfera aproximadamente 750 billones de toneladas de $\mathrm{CO}_{2}$ en este siglo. Debemos recordar que un aumento de $+2^{\circ} \mathrm{C}$ en la temperatura del planeta significaría menos agua y más seca, del $20 \%$ al $30 \%$ de las especies estarían sobre riesgo de extinción, $20 \%$ da Amazona sufriría sabanización, la producción agrícola comenzaría a disminuir en las bajas latitudes y aumentar en las altas latitudes, etc.). En caso de que no se tome ninguna medida, para evitar que esta amenaza natural se realice, serán expelidos hasta el final del siglo aproximadamente 1,4 trillón de toneladas de $\mathrm{CO}_{2}$. Para que se tenga una idea de la magnitud del problema que es evitar la emisión de 750 billones de toneladas de $\mathrm{CO}_{2}$ basta saber que el protocolo de Kyoto, tratado firmado en 1997 para contener los cortes de emisión preveía solamente 5 billones en emisión hasta el 2012.

- Para empeorar el escenario, la segunda parte del informe del IPCC es enfático en relación a la combinación de los cambios climáticos y la acción destructiva del hombre la cual debe superar la capacidad de los ecosistemas de absorber esos impactos y las regiones que más sufrirán serán precisamente las más pobres, por ser regiones donde las 
personas son menos capaces de adaptarse a los cambios climáticos, o sea, el calentamiento convertirá un mundo que ya es desigual en más desigual. (Ver IPCC Working Group II Fourth Assessment Report. Climate Change 2007: Impacts, Adaptation and Vulnerability. Brussels, April 2007.)

El informe destaca tres cuestiones importantes:

- La primera es que de nada valdrá intentar concentrar los cambios solamente en la política de generación de energía, o sea, la industria y la agricultura también tienen que contribuir.

- La segunda se refiere a los costos de cortar las emisiones. Una buena parte del trabajo deberá ser realizada a través de inversiones con retornos y que resultan en costo cero al final, de manera general son las mejoras en la eficiencia energética (ver Tabla 1);

- Por último, y la más complicada cuestión destacada es que las mejores oportunidades para cortar las emisiones están en los países en desarrollo argumentando que "la razón de ese potencial en modo general es que la infraestructura de esos países aún no está totalmente construida", o sea, quien aún esté por construir industrias, centrales, etc., tienen la opción de "seleccionar" o "escoger" tecnologías más limpias. Así para los países que ya tienen completa su infraestructura, como los países desarrollados resultaría mucho más difícil realizar esos cortes.

\begin{tabular}{|c|c|c|}
\hline \multicolumn{3}{|c|}{$\begin{array}{l}\text { TABLA NO. } 1 \text { COSTO DE OPORTUNIDAD "APROXIMADO" DE REDUCIR DE LA } \\
\text { ATMÓSFERA } 40 \text { BILLONES DE TONELADAS DE CO2 (LO QUE REPRESENTA } \\
\text { OCHO VECES LA META DEL PROTOCOLO DE KYOTO) EN 2030) }\end{array}$} \\
\hline GRATIS & ВАJO COSTO & ALTO COSTO \\
\hline 4,2 billones & 10,7 billones & 25 billones \\
\hline \multicolumn{3}{|c|}{ Medidas a ser tomada } \\
\hline $\begin{array}{l}\text { - Cambio de lámparas por } \\
\text { fluorescentes; } \\
\text { - Leyes que prohiban la } \\
\text { producción de carros que } \\
\text { consuman mucho }\end{array}$ & $\begin{array}{l}\text { - Substitución de la } \\
\text { maquinaria industrial; } \\
\text { - Uso de tecnologías } \\
\text { agrícolas menos agresivas. }\end{array}$ & $\begin{array}{l}\text { - Incluyen máquinas para } \\
\text { capturar } \mathrm{CO}_{2} \text { de las } \\
\text { termoeléctricas e inyectarlo } \\
\text { en el subsuelo. } \\
\text { - Desarrollo de tecnologías } \\
\text { como la del carro a hidrógeno. }\end{array}$ \\
\hline
\end{tabular}

Fonte: IPCC Working Group III Fourth Assessment Report. Climate Change 2007: Mitigation of Climate Change. Bangkok - Thailand, May 2007.

Delante de semejante escenario, nada más lógico que los países industrializados intenten eximirse de su responsabilidad histórica en el problema, una vez que son ellos los principales emisores de $\mathrm{CO}_{2}$ y apuestan en revertir esta situación obligando e imponiendo metas a los países menos industrializados. En realidad el problema de la mitigación solamente será resuelto una vez que los principales países emisores asuman sus propias metas, lo que significa en ultima instancia la reducción de sus estándares de consumo y por tanto significaría un cambio radical en sus privilegios, formas y estilo de vida (viciados 


\section{en el uso del vehículo automóvil, específicamente en el transporte individual).}

En el caso de Brasil como atenuante a esta problemática (entendida como la reducción de las emisiones) queda la disminución radical de los "desmatamentos" amazónicos, ya que Brasil es señalado como uno de los países que más emiten $\mathrm{CO}_{2}$ por esa vía.

La preocupación con el medio ambiente entra en la agenda a escala mundial a partir de los años 70, con el reconocimiento de que el modelo de desarrollo productivo del periodo de post guerra había originado problemas de otra naturaleza como: altos niveles de contaminación que comprometían la calidad de vida en general y alto riesgo de agotamiento de los recursos naturales.

Entre los trabajos que más se destacaron a nivel internacional, en la década del 80 fue "Nuestro Futuro Común" de 1987, el cual recoge la idea del desarrollo sostenible y lo define así: "aquel que atiende a las necesidades del presente sin comprometer la capacidad de las futuras generaciones al considerar sus propias necesidades".

En síntesis, el debate mundial sobre el medio ambiente se encaminó a la adhesión de un nuevo estilo de desarrollo que combina eficiencia económica con justicia social y prudencia ecológica, cuyo alcance solamente puede ser viable a través de un esfuerzo conjunto entre los países desarrollados y en vía de desarrollo, o sea, la "llave mágica" para llegar a un futuro mejor se denominó como "desarrollo sostenible".

Esta definición según Moreira (2007), se deriva del paradigma de la globalización, que es de donde provienen esas nociones ambientalistas de rescatar la naturaleza, definición que no hiere los intereses hegemónicos de las naciones, corporaciones nacionales y transnacionales, de las clases sociales, de los dominios territoriales y tecnológicos. Lo que gana estatus es un concepto de "desarrollo sostenible hegemónico", adecuado a la lógica de los mercados globalizados.

En este contexto es cuestionable la forma de cómo obtener un consenso político para construir el futuro que consideramos más adecuado para el planeta si lo que impera es la lógica y la dinámica del capitalismo, que siempre es la obtención del mayor lucro en el menor tiempo posible. En realidad, esas cuestiones sobre lo que se debe hacer empujan cada vez más el proceso de toma de decisiones hacia las decisiones de corto plazo y con un inmediatismo cada vez mayor. O sea, no se trata únicamente de una cuestión de discurso, sino de dominio en una relación de poder. (Ver Moreira, 2007).

\footnotetext{
2 Este estudio también conocido como "Informe Brundtland" denominación derivada del nombre de Gro Harlem Brundtland, primera ministra de Noruega que presidió la comisión encargada por ese tema.
} 
En el caso de Brasil, pensar que el desarrollo sostenible (vía monocultivos como la caña y la soja) va a resolver los problemas del medio ambiente, la desigualdad y la exclusión social es como creer en el Maná, porque el capitalismo es capaz de transformar el medio ambiente y el ecosistema en mercancías para mantener dicho estatus. O sea, bajo la existencia de la propiedad privada y de la ganancia extraordinaria, esa ecología capitalista se apoya en una utopía de mercado que presupone la igualdad de todos como si fuera posible el libre mercado y esa libertad fuese perfecta. En realidad dentro de la orden global contemporánea, bajo el dominio de los oligopolios y de las multinacionales esa libertad no existe. Y ese discurso enmascara (o esconde) los fundamentos sociales y políticos de la desigualdad y esconde las asimetrías de poder (Idem).

Una pregunta es inevitable: ¿por qué es esto importante para discutir la problemática del etanol y los biocombustíbles? Diferentes aspectos deben ser destacados:

a) Con los alertas emitidos en los recientes Informes del IPCC con relación al calentamiento global, los cambios climáticos y la sobrevivencia del globo terráqueo, las mayores economías y las mayores "contaminadoras del planeta" con el pretexto de salvar el medio ambiente (vía mitigación) han lanzado un nuevo negocio altamente lucrativo que amenaza seriamente la producción de alimentos. Se observa que estamos delante una gran campaña global dirigida (por la Unión Europea, EUA y algunos países de América del Sur y Asia) a la producción de biocombustíbles a partir de diferentes materias primas como: la soja, el maíz, el azúcar, el girasol, la palma, la colza, etc., como substitutos derivados del petróleo o por lo menos, como complemento para ser utilizado en los vehículos automóviles. En síntesis esto permitiría cierto amortiguamiento del choque petrolífero en el nivel de precios y simultáneamente, sería una contribución para el combate al omnipresente "calentamiento global". Estos tipos de combustibles son colocados y presentados como "limpios", "biodegradables", "renovables", "verdes" y "sostenibles" y apuntan sobre todo al mercado de los automóviles;

b) En realidad entre los "bastidores del negocio" se está fraguando una alianza inédita en la cual participan las multinacionales petroleras, biotecnológicas, de automóviles y los grandes productores de granos, los cuales decidirán los destinos de las tierras y los paisajes de algunas regiones de América Latina. Debemos recordar que los EUA (el mayor productor de alcohol de maíz del mundo) muestra sus cálculos basados en el mejor de los escenarios de producción irreal de grandes cosechas y de alto aprovechamiento de biocombustíbles, desde su procesamiento hasta la utilización final lo que acabaría por exigir el $121 \%$ de toda la tierra arable del país, para poder producir la biomasa suficiente que substituya el consumo anual de los combustibles fósiles. O sea, para poder sustituir y suplir sus necesidades de petróleo y derivados tendría que cultivar seis veces su superficie.

c) La Unión Europea, en mayo de 2003 adoptó una directiva que promueve el uso de biocombustíbles en los transportes con una previsión de alcanzar una cuota del mercado 
que variaría del 5,75\% al 8\% en el período de 2010 al 2015. En realidad es muy poco probable que esas metas sean alcanzadas según esas proyecciones, ya que exigirá entre $14 \%$ y $19 \%$ de la tierra arable total para que sean dedicadas a la producción de cultivos energéticos, lo que significaría que no quedaría ninguna tierra reservada para proteger la biodiversidad natural, que en la UE es de apenas $12 \%$ del total de la tierra agrícola. Por tanto es evidente que esto será realizado en los países menos desarrollados especialmente en la América Latina, donde ahora argumentan que existen muchos suelos "libres" para el cultivo de la "bioenergía", el sol brilla más durante todo el año y donde las cosechas crecen más rápido, tienen mayor rendimiento y la mano de obra es más barata.

d) Colocado de otra forma, si lo que procuramos son soluciones dentro del paradigma actual de "consumo" creciente de combustible (sea fósil, sea verde) sólo podremos esperar más crisis ambientales y sociales cada vez peores en el futuro. Entendemos que en este sentido el Pico de Petróleo tiene alguna ventaja, como fenómeno que colocará a la humanidad delante de tiempos duros y difíciles, esa ventaja radica en la necesidad de que comprendamos que el paradigma de crecimiento está errado. $\mathrm{O}$ sea, es totalmente equivocado continuar realizando proyecciones y planes económicos para niveles de consumo energético crecientes ignorando que éstos se agotarán durante la primera mitad del actual siglo. Según Rocha (2007) varios especialistas y estudiosos del Pico de Hubbert ${ }^{3}$ comprobaron hasta la saciedad que no existe ningún substituto perfecto para el petróleo o combinación de substitutos que nos permita continuar consumiendo más y más energía cada año que pasa, sea combustible para transporte, sea electricidad, o sea hasta para insumos agrícolas.

Según Alves (2007) delante de esta realidad y sumergidos en un sistema económico caracterizado por la intrínseca necesidad de crecer para vivir, explotando siempre y más intensamente la naturaleza y la humanidad es comprensible que se sientan señales de perturbación y de desorientación en los centros de decisión política. Ya es rutina encontrarnos con toma de decisiones políticas que encierran claras contradicciones entre los objetivos anunciados y los contenidos perseguidos. Esto sucede no por incompetencia de los gobiernos y si debido a la presión de los todopoderosos jefes económicos y financieros que con la gran capacidad que tienen para influenciar a los gobiernos y la experticia que tienen en el arte de sobrevivir, dictan sus recetas para la crisis, con las cuales consiguen "el oro sobre lo azul", o sea, continuarán aumentando sus ganancias (Alves, 2007).

\footnotetext{
${ }^{3}$ Hubbert, es un geofísico, que creo un modelo matemático de la extracción de petróleo y que previo que la cantidad total de petróleo extraída a lo largo del tiempo seguiría una curva logística (inicialmente la función logística tiene un rápido crecimiento, después disminuí hasta que termina por parar). Esto implica que a una determinada altura, la tasa prevista de extracción de petróleo seria dada por la tasa de cambio de la curva logística la cual sigue una curva con la forma de una campana conocida ahora como la curva de Hubbert.
} 


\section{Principales inversionistas extranjeros en la producción de etanol de caña de azúcar en Brasil}

Brasil es el mayor productor mundial en el complejo azucarero ejerciendo el liderazgo en todos los segmentos: caña de azúcar $(39 \%)$, azúcar $(18,4 \%)$ y alcohol $(35,5 \%)$. La producción brasileña de alcohol se expandió a partir del programa de desarrollo para este fin denominado "Proalcool" y de la mezcla obligatoria del producto con la gasolina. Estos hechos sumado a la evolución de las investigaciones científicas proporcionó al Brasil una elevada ventaja competitiva en la producción de azúcar y alcohol.

Los inversionistas extranjeros -que están "atentos" a las ventajas y al potencial de crecimiento del mercado de los biocombustíbles de Brasil (mercado de momento proyectado sin barreras a la entrada de competidores y sin intervención del gobierno, entendido como "sin interferencias directas o indirectas en sus ganancias)"- se dividen en dos tipos: de un lado están los consorcios de empresarios y los fondos de inversión internacionales, interesados en aplicar recursos en un negocio coyuntural, aunque sin involucramiento directo en la operación, del otro lado están las empresas que ya actúan en el sector (de azúcar y alcohol) en el exterior y las tradings que participan o desean participar mas activamente del comercio internacional de etanol. Según esos especialistas, el retorno sobre el capital invertido en este mercado no deber ser menor del $20 \%$ (Ver cuadro 1 ).

\footnotetext{
Fuentes: Elaboración propia a partir de las siguientes informaciones: a) Soros mergulha no etanol. In: Revista Isto é dinheiro. Edição 506. 31/5/2007. b) Alexa Salomão \& Marcelo Onaga. Etanol o mundo quer. O Brasil tem. Em: Revista Exame. 15.06.2006; c) Louis Dreyfus Compra usinas do Grupo Tavares de Melo. En: Ethanol Brasil. Quarta-feira, Fevereiro 21, 2007, d) SISTEMA ECONOMICO LATINOAMERICANO Y DEL CARIBE (SELA). Noble Group centra o foco no Mercosul e estuda aquisições. Tomado de la Selección de Noticias del MRE de Brasil. 12/2/2007. Disponible en: http://www.sela.org/sela/ImpNoticia.asp?id=9414; e) JORNAL FOLHA DE SÃO PAULO. Grupo compra usina no Brasil por US\$ 70 milhões. En: Caderno Dinheiro. 09 de fevereiro de 2007 , f) MÔNICA MAGALHÃES. Noble Group reforça internacionalização do açúcar e álcool e promete ampliar espaço no país. En: JORNALCANA. 26 Mercados \& Cotações. São Paulo Fevereiro/2007. Disponible en: http://www.jornalcana.com.br/pdf/158/\%5Cmerccot.pdf. Acceso; 16-06-2007; g) GESTOR DIGITAL DE INFORMAÇÕES (GDI). Infinity Bio-Energy acerta investimentos no Panamá. 28/05/2007. Disponible en: http://www.eletrosul.gov.br/gdi/gdi/cl_pesquisa.php?pg=cl_abre\&cd=gekdge9;AXfig. Acceso 16-06-2007; h) JORNAL CANA . Infinity Bio Energy incorpora o fundo Evergreen. En: Mercados \& Cotações. Agosto 2006. Disponible en: http://www.jornalcana.com.br/pdf/152/\%5Cmerccot.pdf; Acceso 16-06-2007; i) Informaciones generales del Tereos y la usina Guarani, Disponibles en: http://www.acucarguarani.com.br/br/institucional/Unidades.aspx. J) Brenco instalará quatro usinas no centro-oeste. En: Global Research. 21/5/2007. Disponible en: http://www.globalresearch.com.br/novo/conteudo.asp?conteudo=8435 k) Europeus começam vencendo a guerra. In: Açúcar Ético Disponible en: http://www.sucre-ethique.org/Europeus-comecam-vencendo-a-guerra ; l) JORNALCANA. Fundo internacional planeja investir US\$ 1 bi no Brasil. Agosto/2006. Disponible en: http://www.jornalcana.com.br/pdf/152/\%5Cmerccot.pdf
} 
REVISTA POLIS

VOL. 7 No 21, 2008

PRÒLOGO, págs., 145-165

( ) EIDITORIAL DE LA UNIVERSIDAD BOLIVARIANA DE CHILE, 2008

\begin{tabular}{|c|c|c|}
\hline Cro: & Fati & 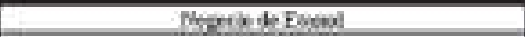 \\
\hline Nokio & 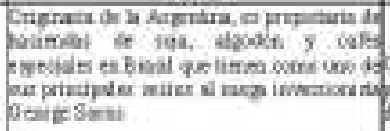 & 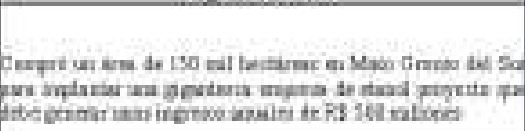 \\
\hline Cand & 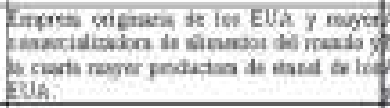 & 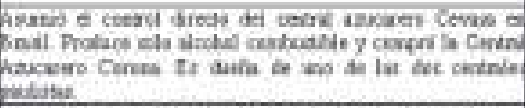 \\
\hline Conis & 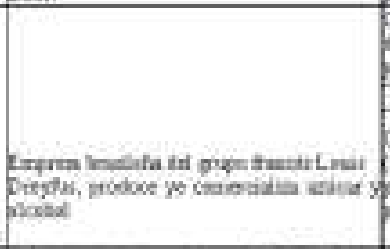 & 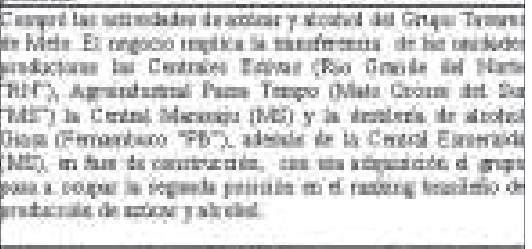 \\
\hline $\begin{array}{l}\text { Now } \\
\text { cirnep }\end{array}$ & 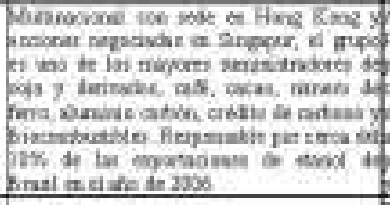 & 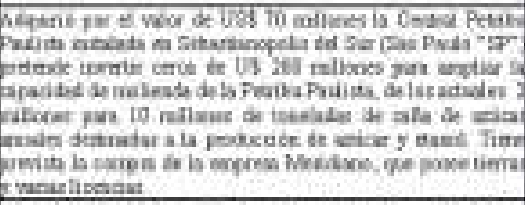 \\
\hline $\begin{array}{l}\ln \ln x y \\
\min \\
\min \end{array}$ & 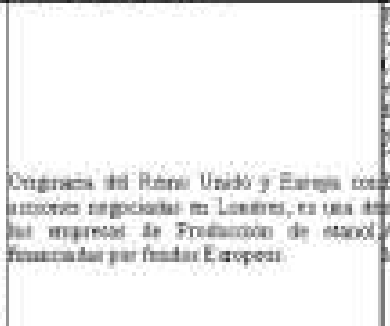 & 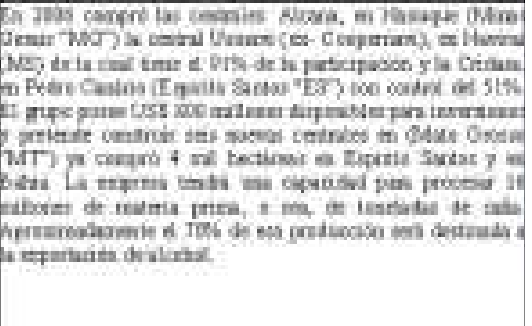 \\
\hline Tana & 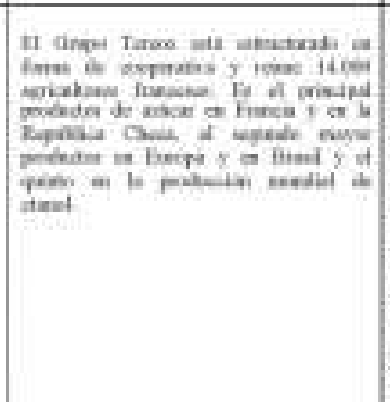 & 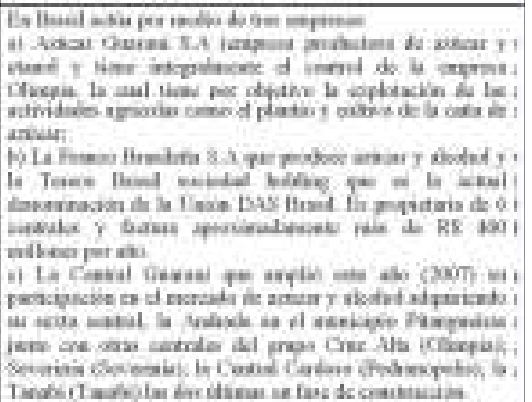 \\
\hline Kitas: & 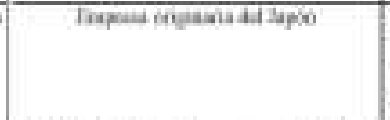 & 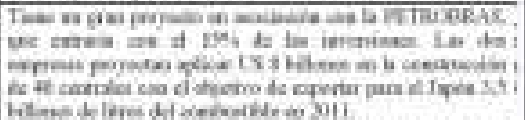 \\
\hline $\begin{array}{l}\text { Ents } \\
00\end{array}$ & 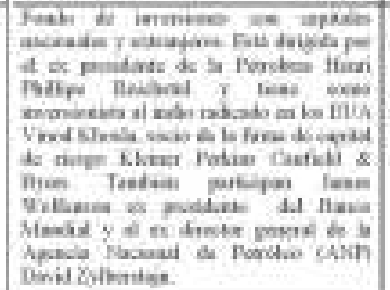 & 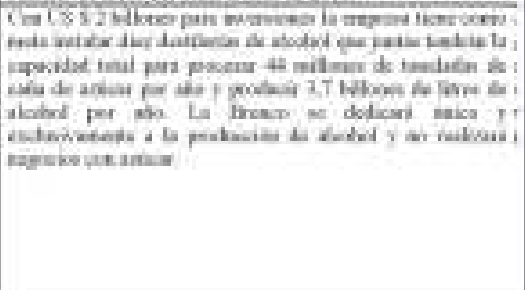 \\
\hline
\end{tabular}

SOSTENIBLE? 
En realidad las macizas inversiones en la construcción de centrales azucareros ya están siendo proyectados. Y Brasil ganaría en promedio una central azucarera por mes en los próximos seis años. Hoy con 336 centrales debe llegar hasta la cifra de 409 al final de la zafra de 2012/2013. O sea, Brasil iría a construir una central por mes hasta el 2013. Además de las 73 centrales confirmados, existen hoy en Brasil 189 consultas abiertas, tanto para la construcción como para la ampliación de las unidades productivas.

Así para colocar a Brasil en el camino de ser el mayor productor de etanol del mundo, el dinero está llegando de todos lados. El Banco Nacional de Desarrollo (BNDES) está decidido a financiar hasta el valor de $\mathrm{R} \$ 10$ billones de la cantidad necesaria para la instalación de nuevas unidades de producción. El resto de los recursos financieros deberá ser aportado por la iniciativa privada nacional e internacional, además de bancos regionales de fomento, como el Banco Interamericano de Desarrollo (BID) y el Japan Bank for International Cooperation (JBIC).

Entre los otros inversionistas extranjeros en el negocio del etanol tenemos al consorcio encabezado por la firma norteamericana Sempra Energy, una de las mayores empresas de gas natural de los EUA y por la firma alemana Manferrostal, del gran grupo Man, que actúa en más de 60 países. El proyecto prevé inversiones de U\$ 8,4 billones en la construcción de 24 centrales productores de alcohol, los cuales exportarán toda su producción dentro de un periodo de 20 años, para esto serán utilizadas 700 mil hectáreas de tierra en Tocantins y otros 800 mil hectáreas ya fueron arrendadas en Maranhão, Mato Grosso y Mato Grosso del Sur, estableciéndose bien próximas de las áreas protegidas de la Amazonia y del Pantanal Mato Grossense. En caso que el grupo alcance la producción deseada (de 5,7 billones de litros anuales) en el año de 2010 produciría el $20 \%$ del total de alcohol producido en Brasil. Los empresarios brasileños del grupo Etalnac administrarían las destilerías y la entrega total de toda la producción de alcohol para la firma Sempra, que a su vez lo exportará para los EUA y el Japón. (Ver, Etanol Brasil, 21-02/2007 Disponible en: http://ethanolbrasil.blogspot.com/2007/02/grupo-alcooleiro-quer-investir-us84.html).

\section{Principales inversionistas extranjeros en la producción de biodiesel en Brasil}

El avance del capital extranjero en los sectores del biodiesel es cada vez mayor y más diversificado. A modo de ejemplo la empresa brasileña Brasil Ecodiesel, la mayor productora de biodiesel en el país, en las subastas de biodiesel realizadas en agosto de 2006, aseguró el suministro del $58,1 \%$ del volumen total a ser comercializado y tiene como 
principal accionista al fondo BT Global administrado por el Deutsche Bank, este en la emisión de acciones en el año 2006, reservó 3,5 millones de sus papeles (recibidos y conocidos por las iniciales ADS) para inversionistas extranjeros. Entre los principales inversionistas extranjeros se destacan. (Ver cuadro 2)

\begin{tabular}{|c|c|c|}
\hline Crupo & Perfil & Negocio en el Biodienel \\
\hline $\begin{array}{l}\text { Global } \\
\text { Energ }\end{array}$ & Empresa originana deE qpaina & 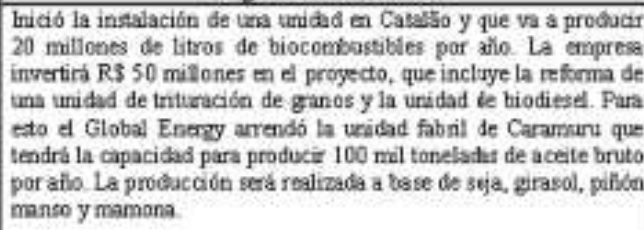 \\
\hline Allbo & 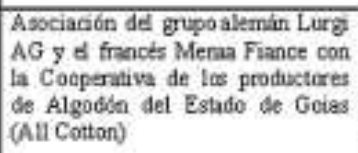 & 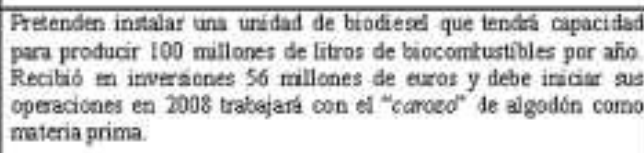 \\
\hline $\begin{array}{l}\text { Archer } \\
\text { Danid } \\
\text { s } \\
\text { Midlan } \\
\text { d } \\
\text { (ADMA) }\end{array}$ & 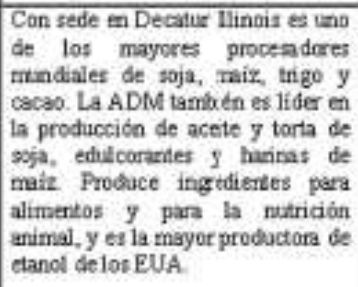 & 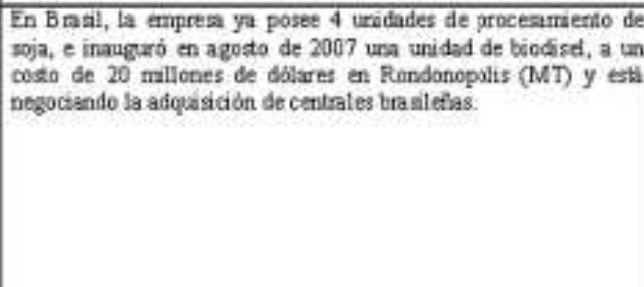 \\
\hline Eunge & 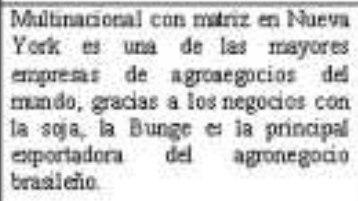 & 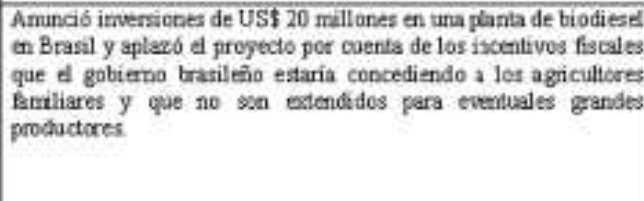 \\
\hline
\end{tabular}

Fuente: Elaboración propia a partir de las siguientes informaciones: a) DIARIO COMERCIO INDUSTRIA \& SERVIÇOS. Capital estrangeiro avança nos setores de etanol e biodiesel. 21/11/2006. Disponible en: http://www.webtranspo.com.br/cargas_noticias2.asp?offset=343\&Registro=11727., b) Informaciones de Brasil Ecodiesel. Disponible en: http://www.brasilecodiesel.com.br/.

Los números son claros y los intereses de las organizaciones financieras por invertir en el mercado de los biocombustíbles en Brasil puede ser visto como una analogía a los bollos financieros de las empresas Ponto con que explotó el final del siglo pasado, o sea, la semejanza estriba en el "capital especulativo", todo es "movido por el capital" y como en todo nuevo mercado aparecen los especuladores, ya que es así como funciona el libre 
mercado y como muy bien argumenta y defiende el empresario Roberto Rodrigues ${ }^{4}$ : “(...) es un mercado sin interferencia del gobierno (...) pues estos grupos financieros y especuladores saben y muy bien lo que hacen y como lo hacen".

Algunas observaciones en relación al mercado de los biocombustíbles (etanol y biodiesel) y las grandes empresas interesadas en invertir en el negocio "lucrativamente sostenible" de Brasil son presentadas a continuación:

- Las empresas multinacionales y los grupos financieros internacionales no participan (y mucho menos responden) en discusiones que envuelvan políticas públicas de desarrollo sostenible, ellas simplemente toman decisiones;

- Sus inversiones son realizadas después de estudios, análisis y reflexiones de carácter sigiloso, análisis que incluyen enfoques dirigidos a la obtención de ganancias extraordinarias en el menor tiempo posible. Esos estudios en la mayoría de los casos no incluyen las cuestiones ambientales y sociales y cuando lo hacen generalmente son presentadas de forma incorrecta;

- Cualquier análisis cuidadoso del sistema capitalista (y de sus multinacionales) rebela que la posibilidad de maximizar la tasa de ganancia siempre depende de no asumir los pasivos sociales y ecológicos, siendo esa la base del sistema y ese es el motivo de que el mundo esté en la situación de crisis global extrema, o sea, los sentimientos que priman en esas empresas son el individualismo y el inmediatismo;

- La gran verdad es que el Brasil no tiene como atender a las demandas del mercado de los EUA y de los otros países "sin desmatar" el Amazonas. El Japón quiere pasar a ser un comprador del producto brasileño pero desea garantías de que el suministro del producto no será interrumpido;

\footnotetext{
${ }^{4}$ Ademas de ser gran productor agrícola, y ex-ministro de la agricultura de Lula, es miembro de un grupo internacional denominado "Comisión Hemisférica de Bioenergía", cuyo objetivo es utilizar la iniciativa privada, junto con los recursos públicos, en un proyecto de producción y comercialización mundial de la energía renovable. Curiosamente, uno de los creadores de la tal comisión Hemisférica, por una increíble coincidencia, es justamente el ex-gobernador de la Florida, Jeb Bush, que también, por increíble coincidencia, es hermano del presidente americano George Bush. Como se ve, los buenos negocios en bioenergía son realizados mezclándose, sin grandes escrúpulos, los intereses estatales y familiares. 
- La expansión de la producción de los biocombustibles es de gran interés para las empresas que producen "Organismos Genéticamente Modificados" como: Syngenta, Monsanto, ${ }^{6}$ Dupont, Dow, Bayer y BASF. En este punto debemos recordar dos cuestiones básicas de principio: la primera es que una de las justificaciones dadas para la existencia de la producción de los OGM consistía en la escasez de disponibilidad de tierras suficientes para combatir el hambre en el mundo, de ahí la necesidad de producir alimentos y cereales (GM) que actualmente son indeseados en casi todo el mundo, fundamentalmente en los países africanos hacia donde los alimentos y las raciones GM están siendo colocadas con el nombre de "ayuda alimentaria" y la segunda que constituye una nueva estrategia y oportunidad de dominación de estas empresas al difundir los productos genéticamente modificados como fuentes de energía "limpia" esperando así menos regulaciones y una mayor aceptación publica, dado que no serán utilizados ni como alimentos ni como raciones. Esto según Mae Wan Ho (2007) expone nuestro ecosistema y los cultivos alimentarios a la contaminación con los cultivos GM que están muy, pero que muy lejos de ser seguros y mucho menos limpios.

- Otros autores como Eric Holt-Gimenez, coordinador de la organización Food First argumenta que se notan dos cuestiones fundamentales dadas las facilidades suministradas por el mercado brasileño de biocombustibles: a) las tres grandes empresas multinacionales (ADM, Cargill y Monsanto) están creando un imperio apoyado en la ingeniería genética; el procesamiento y el transporte, una alianza que pretende amarrar la producción y la venta de etanol y del biodiesel, b) que otras empresas del agronegocio como al Bunge, Sygenta, Bayer y la Dupont aliadas a las transnacionales del petróleo como la Shell, Total, British Petroleum, y a las empresas automotoras como la Volkswagen, Peugeot, Citroen, Renault y SAAB están formando un consorcio inédito que tiene por objetivo la obtención de ganancias extraordinarias con los biocombustíbles y no el desarrollo sostenible de Brasil.

- Las acciones propuestas de coordinación de políticas que hoy tratan el problema de los cambios climáticos a nivel mundial incluido las formas de combatirlas están prácticamente todas en las manos de las empresas multinacionales y de los grupos financieros que conforman el famoso "grupo de los ocho". Acciones que por el bien de la humanidad deberían y deben ser coordinadas y supervisadas por la Organización de la Naciones Unidas como una estrategia mundial de un problema que concierne a todo el planeta.

\footnotetext{
${ }^{5}$ La Syngenta es una empresa transnacional del agronegocio con sede en Suiza. La empresa tiene operaciones en más de 90 países. En 2006, sus vendas alcanzaran el valor de US\$8,1 billones teniendo el 80\% de sus ingresos provenientes de la venda de agrotóxicos y el $20 \%$ de la producción de semillas. La Syngenta es la tercera mayor empresa del sector de semillas del mundo y en el año de 2006 la empresa facturo US\$ 8,1 billones en el mundo.

${ }^{6}$ La Monsanto (multinacional de los EUA) se dedica al desarrollo de tecnologías transgenicas para granos y algodón y creo (en los Estados Unidos, junto con la también americana Cargill) la Renessen. Una joint venture creada para el desarrollo de granos adaptados a la producción de Biocombustíveis. A Renessen recebio inversiones de US\$ 12 millones en la instalación de una planta piloto en el Estado de Iowa. En Brasil, la empresa realiza una asociación con una empresa de capital nacional para desarrollar variedades de caña genéticamente modificada o transgenica. En 2005, la Monsanto facturo en Brasil R \$ 2,251 billones y obtuvo ganancias liquidas de $\mathrm{R} \$ 182,354$ millones, contra $\mathrm{R} \$ 18,521$ millones en el año anterior. (Ver, http://www.jornaldaciencia.org.br/Detalhe.jsp?id=42947) 


\section{Consideraciones finales}

En realidad el desafío lanzado a la humanidad a través de los Informes del IPCC de sustituir (y rápidamente) la dependencia de fuentes de energía no renovables por alternativas verdaderamente sostenibles coloca en jaque "a la sabiduría tradicional" fundamentalmente aquella promovida por las transnacionales y multinacionales dominantes, con relación a la creencia absoluta en el libre mercado como la mejor fuente de innovación y aprendizaje para desarrollar alternativas energéticas viables y sostenibles ambientalmente

El trabajo llama la atención en esa cuestión ya que el libre mercado ignora soluciones que no puedan resultar en ganancias extraordinarias. Cualquier empresa o firma que deje de seguir este simple principio no permanecerá en el negocio por mucho tiempo, lo que vale también para las empresas y firmas productoras de biocombustibles. O sea, el libre mercado ignora cualquier solución que no pueda ser controlada, sea mediante los intereses de propiedad (propiedad intelectual forzosa, licencias de monopolio, etc.) sea con la centralización exigida por las economías de escala. Esto significa que el libre mercado es estructuralmente incompatible con una enorme porción del universo de posibles soluciones energéticas.

El libre mercado gusta de las fuentes de energía no renovables porque ellas son fácilmente controladas. En países donde los derechos sobre los minerales son propiedad privada (como en los EUA y Canadá) estos recursos pueden ser controlados vía derechos de propiedad. En el resto del mundo, ellos pueden igualmente ser controlados fácilmente a través de contratos exclusivos con los gobiernos.

En el caso de la producción de biocombustíbles, esto no es diferente para las grandes multinacionales que intentan "adaptar y utilizar" su herramienta habitual la propiedad a la solución del problema. Si tomamos como ejemplo el etanol y los otros biocombustíbles brasileños, esta tentativa de solución para nuestros problemas energéticos están siendo controlados a través de la propiedad o del arrendamiento real de tierras que producen las materias primas y/o con la propiedad intelectual (procesos propietarios de destilación, microbios patentados que convierten substancias en azúcares, etc). O sea, no importa que los biocombustíbles proporcionen un bajo retorno energético sobre la inversión o que ellos creen más problemas fundamentales como el crecimiento en curso de la demanda de energía, agotamiento de los suelos fértiles, la contaminación o la competencia entre los alimentos y la energía. Ellos pueden hacer mucho dinero y es esto lo que importa.

En relación con la preservación ambiental cualquier camino efectivo que lleve a una reducción del consumo de energía no renovable choca con la misma dificultad: la disminución de la ganancia o de los lucros extraordinarios, lo que negaría la esencia del libre mercado. 
Debemos dejar claro que el libre mercado "puede" ayudar a innovar alguna cosa para vender (como por ejemplo los biocombustibles) que lo ayudará a "conservar" no obstante el acto real de preservación ambiental mata los lucros. Esto, como muy bien lo explica Jeff Vail, no se debe confundir con la eficiencia incrementada por el uso de la energía (la cual, como explica la ciencia económica clásica, reduce los costos y libera al consumidor para gastar el dinero ahorrado en consumo, elevando por tanto el estándar de vida total, cuando éste es medido en función del consumo). En el trabajo estamos haciendo referencia a la preservación ambiental real, aquella que simplemente significa: utilizar menos.

Esta vía de hecho es un pensamiento maldito para la ciencia económica del libre mercado, la idea de que podríamos utilizar menos energía en total y así invertir los ahorros en bienes no económicos (tales como tiempo de descanso, seguridad, salud, educación, etc.) mediante la auto suficiencia es altamente problemático porque ella causaría una disminución acumulativa en el PIB y en los estándares de consumo (el tiempo de descanso no cuenta como un producto o mercancía). Es como si un gerente de una firma comentara: ¡Aquí está mi plan de negocios, no pretendo vender nada y cuando todo fuera dicho y hecho, las personas nos utilizarán menos. Vamos a ser ricos! (Jeff Vail, 2007).

Al confiarse en el libre mercado como la solución del problema energético en un mundo en que la producción fósil esta a punto de alcanzar el pico, existiría apenas un resultado garantizado: sus soluciones jamás nos liberarán de la dependencia energética o de la escasez de energía. Esto significa o equivale a decir que el libre mercado nunca producirá una solución para este problema porque los consumidores dependen de las firmas para los productos que ellos compran y es así como se produce la ganancia o el lucro. Análogamente el libre mercado nunca tendrá la motivación económica para tornar la energía más barata (a largo plazo) esto sería por definición un comportamiento económico irracional producir la energía de forma tan barata que su valor total de mercado mundial se viniese abajo.

Por tanto podemos afirmar que el éxito de los biocombustibles dependerá de su uso obligatorio, de las facilidades fiscales, de los subsidios suministrados por el Estado, de la tasación a los consumidores, del desconocimiento de los derechos de los trabajadores y de las mil y unas formas de arrancar de las comunidades rurales el uso real y efectivo de sus tierras. 


\section{Bibliografía}

Açúcar 'etico (2007), Europeus começam vencendo a guerra. In: Açúcar Ético Disponível In: http://www.sucre-ethique.org/Europeus-comecam-vencendo-a-guerra . Acesso 16-06-2007.

Alexa Salomão \& Onaga M. (2006), «Etanol o mundo quer. O Brasil tem». In: Revista Exame. 15.06.2006.

Alves D. (2007), Bio-confusão. Disponível In: http://resistir.info/energia/bioconfusao.html. Acesso 12/09/2007.

Asociacão Nacional dos Fabricantes de Veiculos Automotores (2007), In: Estatísticas. Disponível In: http://www.anfavea.com.br/tabelas.html. Acesso $12 / 09 / 2007$.

Attuch L. (2007), «Soros mergulha no etanol». In: Revista Isto é dinheiro. Edição 506. 31/5/2007.

Benoit H. (2007), Aliados na miséria e na barbárie mundial 2007. Disponível In: http://www.wsws.org/pt/2007/mar2007/port-m09.shtml. Acesso 16-06-2007.

Borón A. (2007), Biocombustibles: el porvenir de una ilusión. Disponivel In: http://www.rebelion.org/noticia.php. Acesso 16/06/2007.

8. Brasil Ecodiese (2007), Informações da Brasil Ecodiesel, disponível In: http://www.brasilecodiesel.com.br/. Acesso 16-06-2007.

Carpintero O. (2006), «Biocombustibles y uso energético de la biomasa un análisis critico». In: Revista El ecologista, $\mathrm{n}^{\circ}$ 49, 2006.

Carvalho C. (2007), «Para onde vai o etanol brasileiro?». In: Portal do Mundo do Trabalho. Disponível In: http://www.cut.org.br/site/print.php?infoid=10547\&sid=21. Acesso 31-05-2007.

Carvalho, G.R. (2006), O setor sucroalcooleiro em perspectiva. Boletim de conjuntura agropecuária. Campinas: Embrapa Monitoramento por Satélite, março de $2006 . \quad$ Disponível In: http://www.cnpm.embrapa.br/conjuntura/0603_Sucroalcooleiro.pdf. Acesso em: $11 / 04 / 2007$

Cortez H. (2007), Acordo Brasil e EUA sobre o etanol: O desastre que nos espera. Disponível In: http://www.carbonobrasil.com/article.htm?id=125490. Acesso 16-062007. 
Diario Comercio Industrias \& Serviços (2007), Capital estrangeiro avança nos setores de etanol e biodiesel. 21/11/2006. Disponível In: http://www.webtranspo.com.br/cargas_noticias2.asp?offset=343\&Registro=11727. Acesso 16-06-2007.

EMBRAPA. Mapa da Cobertura Vegetal do Brasil. Disponível In: http://www.cobveget.cnpm.embrapa.br/resulta Acesso 31-05-2007.

FAO. El Estado Mundial de Agricultura y la Alimentación 2005. Disponível In: http://www.fao.org/docrep/008/a0050s/a0050s15.htm\#C5. Acessado 10/09/2007.

Gestor Digital de Informações(GDI) (2007), Infinity Bio-Energy acerta investimentos no Panamá. 28/05/2007. Disponível In: http://www.eletrosul.gov.br/gdi/gdi/cl_pesquisa.php?pg=cl_abre\&cd=gekdge9;AXfig . Acesso 16-06-2007.

Goldemberg J. (2007), «Ethanol for a Sustainable Energy Future». In: Science Vol. 315. no. 5813, 9 February 2007, pp. $808-810$.

Guarani (2007), Informações gerais do grupo Tereos e a usina Guarani, Disponíveis In: http://www.acucarguarani.com.br/br/institucional/Unidades.aspx. Acesso 16-062007.

Instituto Brasileira de Geografi e Estatistica (IBGE) (2007), Levantamento sistemático da produção agrícola para 2003-2004; http://www.sidra.ibge.gov.br, Acesso 12/09/2007.

Intergovernmental Panel on Climate Change Working (IPCC) (2007), Working Group III Fourth Assessment Report. Climate Change 2007: Mitigation of Climate Change. Bangkok - Thailand, May 2007.

Idem (2007), Working Group II Fourth Assessment Report. Climate Change 2007: Impacts, Adaptation and Vulnerability. Brussels, April 2007.

Idem (2007), Group I to the Fourth Assessment Report of the Climate Change 2007: The Physical Science Basis, February, 2007.

Jank, M. (2007), Dinâmica e perspectiva dos Biocombustíveis no Brasil e no mundo. São Paulo, 8 de março de 2007. Disponível in: http://www.iconebrasil.com.br/pt/. Acesso 31-05-2007.

Jornal Cana (2007), Infinity Bio Energy incorpora o fundo Evergreen. In: Mercados \& Cotações. Agosto 2006. Disponível in: http://www.jornalcana.com.br/pdf/152/\%5Cmerccot.pdf. Acesso 16-06-2007. 
Jornal Folha de São Paulo (2007), Grupo compra usina no Brasil por US\$ 70 milhões. In: Caderno Dinheiro. 09 de fevereiro de 2007.

Jornal O Estado de São Paulo (2007), Etanol Brasil, 21-02/2007 Disponível In: http://ethanolbrasil.blogspot.com/2007/02/grupo-alcooleiro-quer-investir-us84.html). Acesso 16-06-2007.

Jornal Valor (2007), Brenco instalará quatro usinas no centro-oeste. In: Global Research. 21/5/2007. Disponível In: http://www.globalresearch.com.br/novo/conteudo.asp?conteudo=8435. Acesso 1606-2007.

Jornalcana (2007), Fundo internacional planeja investir US\$ 1 bi no Brasil. Agosto/2006. Disponível In: http://www.jornalcana.com.br/pdf/152/\%5Cmerccot.pdf. Acesso 16-06-2007.

Lozza A. (2007), ¿Bio o business? Disponível In: http://www.rebelion.org/noticia.php?. Acesso 16-06-2007

Mae-Wan Ho (2007), Biocombustíveis Biodevastação fome \& falsos créditos de carbono. Disponível In: http://www.i-sis.org.uk/BiofuelsBiodevastationHunger.php

Magalhães Mônica (2007), «Louis Dreyfus Compra usinas do Grupo Tavares de Melo». In: Ethanol Brasil. Fevereiro 21, 2007. Disponível In: http://ethanolbrasil.blogspot.com/2007/02/louis-dreyfus-compra-usinas-dogrupo.html. Acesso 31/05/2007.

Idem (2007), «Noble Group reforça internacionalização do açúcar e álcool e promete ampliar espaço no país». In: Jornalcana. 26 Mercados \& Cotações. São Paulo Fevereiro/2007. Disponível In: http://www.jornalcana.com.br/pdf/158/\%5Cmerccot.pdf. Acesso; 16-06-2007.

Mercado \& Negocios (2006), «Biodiesel primeiros passos». In: Revista Agroanalysis da FGV. No. 9. Vol. 26. Set. 2006.

Monbiot G. (2007), «Uma solução letal». In: Informação Alternativa. 27 de março de 2007 Disponível http://infoalternativa.org/autores/monbiot/monbiot047.htm Acessado 14-06-2007.

Moreira R. (2007), Desenvolvimento sustentável é possível no capitalismo? Disponível In: http://www.faperj.br/boletim_interna.phtml?obj_id=3575. Acesso 12/09/2007.

Nellemann, C., Miles, L., Kaltenborn, B. P., Virtue, M. \& Ahlenius H. (2007), UNEP \& UNESCO, (Eds). The Last Stand of the Orangutan. State of Emergency: Illegal 
Logging, Fire and Palm Oil in Indonesia's National Parks (pdf). 10 de Fevereiro de 2007.

Patrícia Aparecida Ferreira et all. (2006), Governança Corporativa e o Novo Mercado: uma estratégia de capitalização para o setor sucroalcooleiro. Disponível In: http://www.ead.fea.usp.br/Semead/9semead/resultado_semead/trabalhosPDF/326.pdf. Acessado 10/09/2007.

Paulillo L. \& Vian F. C. (2005), «Análise da Competitividade das Cadeias de Agroenergia no Brasil». In: BuanaimM, A. M.; Batalha, M. O. Análise da competitividade das cadeias agroindustriais brasileiras. Projeto MAPA/IICA UFSCar/UNICAMP 2005.

Pimentel, D (2003), "Ethanol fuels: energy balance, economics and environmental impacts are negative", Natural Resources Research, 12, pp. 127-134;

Pimentel, D., T.W. Patzek (2005), "Ethanol Production Using Corn, Switchgrass, and Wood; Biodiesel, Production Using Soybean and Sunflower", Natural Resources Research, 14, pp. 65-76.

Rocha, L. (2007), Etanol e Biocombustíveis Problemas Sociais, Problemas Ambientais e Eficiência Energética. Disponível In: http://odiario.info/articulo.php?p=246\&more=1\&c=1. Acesso 12/09/2007.

Rofrigues, A. (2005), «Etanol Combustível: Balanço e Perspectivas». In: Única. Campinas, 16 de novembro de 2005.

Salomão, A. (2006), «A revolução que vem de fora». In: Revista Exame, ed. 862, n.4, p. 28 -32, Março de 2006.

Sistema Economico Latinoamericano y del Caribe (SELA) (2007), Noble Group centra o foco no Mercosul e estuda aquisições. Tomado de la Selección de Noticias del MRE de Brasil. 12/2/2007. Disponível In: http://www.sela.org/sela/ImpNoticia.asp?id=9414. Acesso; 16-06-2007.

Tetti, Laura (2005), «A Experiência dos Produtores de Etanol». In: Macedo Isaias (org). A Energia da Cana-de-Açúcar. ÚNICA. 2005.

VAIL, J. (2007), Porque o mercado livre engana os consumidores quanto à inovação energética sustentável. Disponível In: http://ww w.jeffvail.net Acessado 10/09/2007.

Wetlands International, Bio-fuel less sustainable than realised, 08/12/2006 